\title{
Onset of Ferrielectricity and the Hidden Nature of Nanoscale Polarization in Ferroelectric Thin Films
}

\author{
Matías Nuñez ${ }^{1}$ and M. Buongiorno Nardelli ${ }^{1,2}$ \\ ${ }^{1}$ Center for High Performance Simulation and Department Physics, North Carolina State University, \\ Raleigh, North Carolina 27695, USA \\ ${ }^{2}$ CCS-CSM, Oak Ridge National Laboratory, Oak Ridge, Tennessee 37831, USA
}

(Received 13 March 2008; published 5 September 2008)

\begin{abstract}
Using calculations from first principles and the concept of layer polarization, we have elucidated the nanoscale organization and local polarization in ferroelectric thin films between metallic contacts. The profile of the local polarization for different film thicknesses unveils a peculiar spatial pattern of atomic layers with uncompensated dipoles in what was originally thought to be a ferroelectric domain. This effectively ferrielectric behavior is induced by the dominant roles of the interfaces at such reduced dimensionality and can be interpreted using a simple classical model where the latter are explicitly taken into account.
\end{abstract}

PACS numbers: 77.80.Dj, 31.15.es, 77.55.+f, 77.90.+k

Since ferroelectricity is a macroscopic collective property of bulk materials, its existence in ultrathin nanoscale films is a matter of debate and a subject of intense scientific scrutiny $[1,2]$. In a seminal theoretical paper, Junquera et al. [3] have suggested that the appearance of ferroelectricity in thin films of $\mathrm{BaTiO}_{3}$ with $\mathrm{SrRuO}_{3}$ contacts should be limited to thicknesses of more than six unit cells. More recently this picture has been broadened, and a variety of studies have shown that thin oxide films can indeed maintain some ferroelectricity at thicknesses even smaller than six unit cells [4-7].

The size limit of ferroelectricity in ultrathin films can be understood using a simple electrostatics argument: as the thickness of the oxide film is reduced, the intensity of the depolarization field (DF) is made stronger [3], thus increasing the electrostatic energy of the system. In a ferroelectric crystal this energy contribution can be minimized in two ways: either by breaking the polarization pattern in $180^{\circ}$ stripe domains to reduce the magnitude of the surface dipole density or by a reduction of the ionic polarization while the system remains in a monodomain state [7]. The final polarization pattern depends on the individual material and/or structure, and understanding which system modification will occur under what conditions is still a matter of debate [7].

At small dimensions, the spatial confinement and the interface details become extremely relevant since they determine the spatial localization of the electrons and thus influence directly the ferroelectric characteristics and the electronic properties of the system [8]. Therefore, to clarify the behavior of ferroelectric thin films in the nanometer regime it is crucial to obtain a precise description not only of the geometry and electronic properties of the film but first and foremost of the polarization profile inside the oxide.

In order to obtain a complete description of ferroelectricity at the nanoscale we have exploited the notion of layer polarization (LP). This idea, based on modern theory of polarization $[9,10]$ and the concept of maximally localized Wannier functions [11], was recently introduced to describe the layer-by-layer modulation of polarization in ferroelectric superlattices [12-14].

We have applied this method to a model metalferroelectric system $\left(\mathrm{BaTiO}_{3} / \mathrm{Pt}\right)$ and obtained a detailed spatial profile of the polarization in the oxide region in the direction of growth that accounts not only for the ionic displacements but also for the spatial rearrangement of the electron density. It is important to note that using more standard methods to evaluate macroscopic polarization, this information would have remained hidden by the averaging procedure, or would have produced misleading results.

In this Letter we show that the ferroelectric structures associated with small length scales are more complex than previously thought, mainly due to the redistribution of the electrons under the constraints imposed by the interfaces. In particular, the oxide develops a ferrielectric pattern of an unbalanced dipole structure that is particularly evident in Fig. 1(a), where we show how positive ionic displacements (rumplings) on all the atomic layers of a thin $\mathrm{BaTiO}_{3}$ film correspond actually to layer dipoles of opposite signs. This is a consequence of the interplay between the orientation and magnitude of the dipoles with the DF, their mutual interaction and the nature of the interfaces. A simple analytic model of a ferroelectric thin film where these effects are explicitly taken into account can capture all these features and it will be discussed in detail at the end.

We have simulated thin films of $\mathrm{BaTiO}_{3}$ between $\mathrm{Pt}$ metal contacts in a (001) stack, where the oxide is terminated with a $\mathrm{BaO}$ plane at both interfaces [15] and the system is simulated under short-circuit boundary conditions. The different supercells can be labeled as $\mathrm{Pt} /\left(\mathrm{BaO}-\mathrm{TiO}_{2}\right)_{m}-\mathrm{BaO} / \mathrm{Pt}$ with $m=1,2,4,6$. We used 


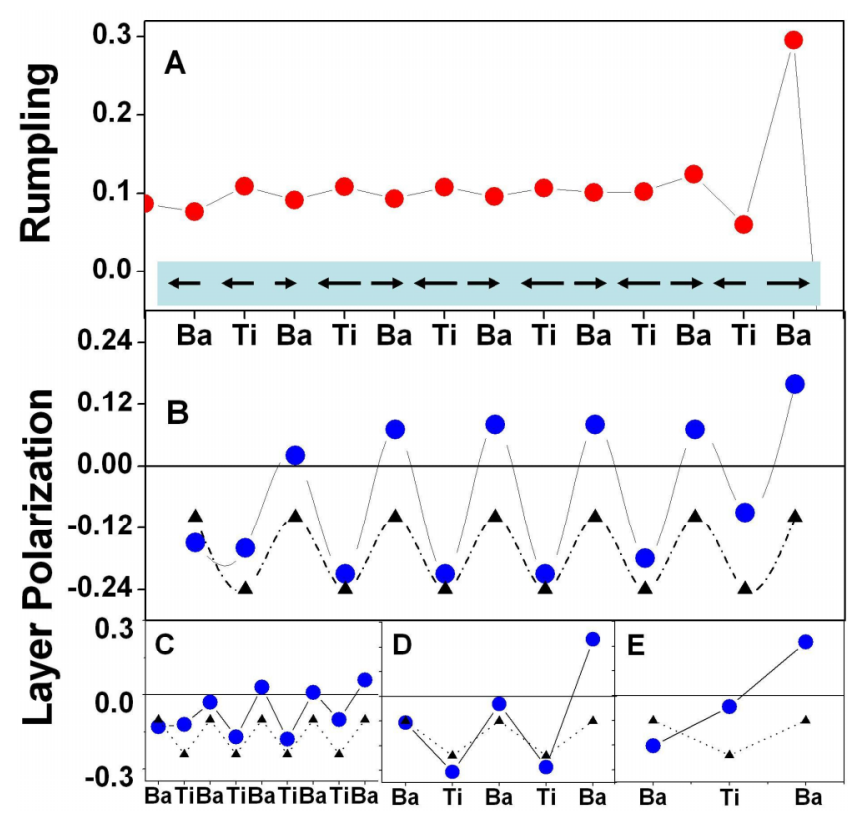

FIG. 1 (color online). (a) Rumpling [cation-oxygen perpendicular distance (in $\AA$ ) within each atomic layer] profile for six unit cells $(m 6)$ of $\mathrm{BaTiO}_{3}$ sandwiched between Pt contacts. (On the horizontal axis $\mathrm{Ti}$ and $\mathrm{Ba}$ indicate the position of the individual $\mathrm{TiO}_{2}$ and $\mathrm{BaO}$ planes, and the orientation of the individual layer dipoles is displayed by arrows in the bottom of the panel.] (b) Solid (blue) circles: layer polarization (LP) profile for the same system $m 6$ in units of $10^{-10} \mathrm{C} / \mathrm{m}$. The values for bulk $\mathrm{BaTiO}_{3}$ are shown with black triangles. (c)(e) LP profiles for $m 4, m 2$, and $m 1$, respectively.

the experimental in-plane lattice parameter of $\mathrm{BaTiO}_{3}$ $(3.991 \AA)$, and kept it fixed in all calculations while all other geometrical parameters were fully relaxed. All simulations have been performed using density functional theory within the local density approximation, with ultrasoft pseudopotentials and a plane waves basis set [16].

Following Ref. [14], we write the polarization of a single atomic plane (LP) as

$$
p_{j}=\frac{1}{A} \sum_{\tau \in j} Q_{\tau} \vec{R}_{\tau z}-\frac{2 e}{A} \sum_{m \in j} \vec{r}_{m z}
$$

where $j$ defines the layer, $\tau$ and $m$ run, respectively, over ion cores (of charge $Q_{\tau}$ located at position $\vec{R}_{\tau}$ ) and the Wannier centers of charge $2 e$ (located at $\vec{r}_{m}$ ), belonging to layer $j . A$ is the in-plane area of the cell, and we are only interested in the $z$ components (parallel to the growth direction). The Wannier centers have been obtained using the maximally localized Wannier functions (MLWFs) algorithm originally proposed by Marzari and Vanderbilt [11] as implemented in the WANT code [17]. Since the valence bands of the oxide and the bands of the metal are mixed in the full supercell calculation, a disentanglement procedure $[17,18]$ was applied before starting the localization algorithm.

The set of MLWFs so obtained cluster around each atomic layer in sets containing the correct number of
Wannier function needed to keep the layer charge neutral. This is a crucial condition for the validity of the definition of LPs in Eq. (1) [14] and allows us to associate each set of Wannier centers to the corresponding crystal plane without ambiguity. Moreover, we can associate each LP value $p_{j}$ with its correspondent (dimensionally correct) dipole $p_{j} A$, which gives precise information about the orientation and magnitude of the atomic layer dipoles [19].

The LP profile calculated for the metal-oxide systems provides a detailed local description of the polarization, which is superior and even contradictory to the information obtained from empirical evaluations based solely on ionic displacements. As an illustration, in Fig. 1(a) we display the rumpling profile for a thin film comprised of six $\mathrm{BaTiO}_{3}$ unit cells $(m 6)$. From the displacement profile one would be tempted to conclude that the film is in a ferroelectric domain configuration, since the rumpling values (and hence the "classical" local dipoles) associated to each plane are positive. Instead, if the correspondent LP profile is analyzed, a completely different picture emerges, where the main feature is an alternation of positive and negative values of the LP in the individual atomic layers of the oxide film. This is clearly shown in Fig. 1(b), where we plot the LP's values computed for $m 6$. Here the central $\mathrm{BaO}$ planes have positive dipoles while the $\mathrm{TiO}_{2}$ planes have negative ones. The first are aligned with the direction of the DF, while latter oppose it. A schematic illustration of the associated dipoles is drawn above the plot.

This result implies that what could have been interpreted as a ferroelectric domain using standard geometrical information in fact displays a much more complex spatial pattern of polarization. We believe that this effect is comparable to the onset of the formation of two-dimensional domain islands commonly observed both theoretically and experimentally in ferroelectric films [7]. In that case, the domains are composed of adjacent $180^{\circ}$ domains in order to minimize the electrostatic energy associated with the DF. Here, our results show that the system can reduce its electrostatic energy in an alternative way: the energetic interplay between the dipoles, the DF, and the interface bonding drives the individual atomic layer dipoles to arrange themselves in an uncompensated dipole pattern along the growth direction [see inset of Fig. 1(a)], or, in other words, the system undergoes a ferroelectric-toferrielectric phase transition.

Moreover, we also predict the existence of a critical thickness above which the system exists in a true ferroelectric domain with all the dipoles pointing in the same direction [see Fig. 1(b), where the LP's values for bulk $\mathrm{BaTiO}_{3}$ are shown with triangles].

As the thickness is reduced, the magnitude of all the dipoles is diminished by the increase of the DF that opposes them. Because of the different physical properties of consecutive layers $\left(\mathrm{BaO}\right.$ and $\left.\mathrm{TiO}_{2}\right)$, their associated dipoles reduce their magnitude at different rates. At the critical thickness, the $\mathrm{BaO}$ layers will have null dipoles and 
eventually they will flip layer polarization direction, aligning with the DF in order to minimize the associated internal energy and creating a ferrielectric dipole pattern (FDP).

The formation and characteristics of the FDP are determined by the bonding properties of the interfaces that pin the LP values of the outer layers of the oxide film. At small film thicknesses the interface effects become dominant and induce the overall spatial variation of the LPs that determines the orientation and magnitude of the layer dipoles along the structure.

We can see how the FDP observed in the $m 6$ geometry is increasingly influenced by the locality of the interfaces as the thickness is reduced to $m 4, m 2$, and $m 1$ [see Figs. 1(c)1(e), respectively]. The central planes become more and more influenced by the interface environment, while the FDP becomes irregular, and finally disappear when the film is comprised by just 3 layers (i.e., one unit cell, $m 1$ ), leaving instead a centrosymmetric paraelectric structure [Fig. 1(e)]. It is important to note that exclusively electronic effects drive this series of regime transitions. In fact, the ionic polarization, directly proportional to the ionic rumpling, remains almost constant for all the thicknesses considered, and it is only the electronic polarization that changes its values in the different systems.

As the size of the film is increased, the DF decreases and the dipoles that are oriented along the DF direction get smaller, while the interface effects lose relevance. At the critical size, the dipoles flip direction and a ferroelectric domain structure is established again. Eventually, the dipoles will relax to the bulk structure, shown with triangles in Fig. 1.

We further illustrate these physical principles by developing a simple classical model of a ferroelectric thin film that reproduces the appearance of a FDP. We extended the model introduced in Ref. [3] by discretizing the thin film along the growth direction in each of its component unit cells in order to introduce a local spatial description of the polarization with the introduction of the local polarization, $P_{i}$. Each individual crystal unit cell is composed of two consecutive layers of $\mathrm{BaO}$ and $\mathrm{TiO}_{2}$; therefore, $P_{i}$ could be obtained by adding each individual LP and dividing by the lattice constant in the stacking direction $c$. The full polarization profile of the film is completely specified by the full set of LPs $\left(p_{1}, p_{2}, \ldots, p_{2 M-1}, p_{2 M}\right)$ associated with each crystal plane of the oxide film.

We can write the total energy of the thin film composed by $M$ unit cells as

$$
\begin{aligned}
E= & \sum_{i=1}^{M}\left(-a P_{i}^{2}+b P_{i}^{4}\right)-\sum_{i=1}^{M} \Omega \vec{P}_{i} \cdot \vec{E} \\
& -\frac{A^{2}}{8 \pi \varepsilon} \sum_{i, j=1}^{2 M} \frac{\theta_{i j} p_{j} p_{i}}{d_{i j}^{3}},
\end{aligned}
$$

where each term of the first sum accounts for the internal energy of the individual unit cells under zero electric field. The parameters $a$ and $b$ are obtained from an $a b$ initio calculation of the total energy of $\mathrm{a} \mathrm{BaTiO}_{3}$ bulk for different ionic displacements along the soft mode [20]. The contribution from the depolarization field $\vec{E}$ is included in the second sum [3], where $\Omega$ is the volume of the unit cell. Each one of its terms favors energetically the local dipoles that follow the same direction as the DF. The third sum represents the classical electrostatic energy for a series of dipoles separated by a distance $d_{i j}$, and can be separated in three contributions: two that account for the interaction between the interface dipoles with the internal ones, and another one that accounts for the mutual electrostatic interaction between the internal dipoles. We have chosen to assign a fixed magnitude and direction to the interface layer dipoles $\left(p_{L}\right.$ and $\left.p_{R}\right)$, with two distinct interaction parameters $\theta_{L}$ and $\theta_{R}$, thus establishing the locality of the interface environment [21]. The interaction parameter $\theta_{i j}$ for the internal dipoles is arbitrarily chosen to be unity.

With the constraint imposed by the simplicity of the model, we look for the energetically more favorable spatial polarization profile as a function of the film thickness. Since the total energy of the system can be expressed as a function of only two single variables $p_{\mathrm{BaO}}$ and $p_{\mathrm{TiO}_{2}}$, the spatial profile of polarization that characterize the film can be expressed as the vector $\left(p_{L}, p_{\mathrm{TiO}_{2}}, p_{\mathrm{BaO}}, p_{\mathrm{TiO}_{2}}, \ldots\right.$, $\left.p_{\mathrm{BaO}}, p_{\mathrm{TiO}_{2}}, p_{R}\right)$ where a ferroelectric domain and a FDP will have all dipoles parallel or antiparallel, respectively. Note that the total energy per unit cell $\left(E^{\prime}=E / M\right)$ can be expressed as a function of only two single variables $p_{\mathrm{BaO}}$ and $p_{\mathrm{TiO}_{2}}$ in the form

$$
\begin{aligned}
E^{\prime}\left(p_{\mathrm{TiO}_{2}}, p_{\mathrm{BaO}}\right)= & -a\left(\frac{p_{\mathrm{TiO}_{2}}+p_{\mathrm{BaO}}}{c}\right)^{2}+b\left(\frac{p_{\mathrm{TiO}_{2}}+p_{\mathrm{BaO}}}{c}\right)^{4}+\Omega E\left(\frac{p_{\mathrm{TiO}_{2}}+p_{\mathrm{BaO}}}{c}\right) \\
& -\frac{A^{2}}{4 \pi \varepsilon}\left(\frac{\alpha p_{\mathrm{TiO}_{2}}}{M c^{3}}+\frac{p_{R} p_{L}}{M(2 M-1) c^{3}}+\frac{1}{M} \sum_{i=3}^{2 M-1} \frac{p_{L} p_{i}}{d_{L j}^{3}}+\frac{1}{M} \sum_{i=3}^{2 M-1} \frac{p_{i} p_{R}}{d_{i R}^{3}}+\frac{1}{2 M} \sum_{i, j=1}^{2 M-2} \frac{p_{j} p_{i}}{d_{i j}^{3}}\right),
\end{aligned}
$$

where we define an overall interface parameter $\alpha=\left(\theta_{R} p_{R}+\theta_{L} p_{L}\right)$.

The thickness of the film is determined by the number of unit cells $M$ and directly affects the magnitude of the depolarization field $E$. The DF is calculated from an ab initio calculation for a supercell with the same size $M$ and using a well-established averaging technique for the electrostatic potential inside the film [21].

As $M \rightarrow \infty$, the terms in Eq. (3) that describe the mutual interaction between the dipoles converge to constant values, the DF vanishes, and the model recovers the usual bulk properties. Only for small $M$ the dipolar terms contribute appreciably to the total energy of the system. This is true, in particular, for the interface term that carries the information that defines the interfaces. 


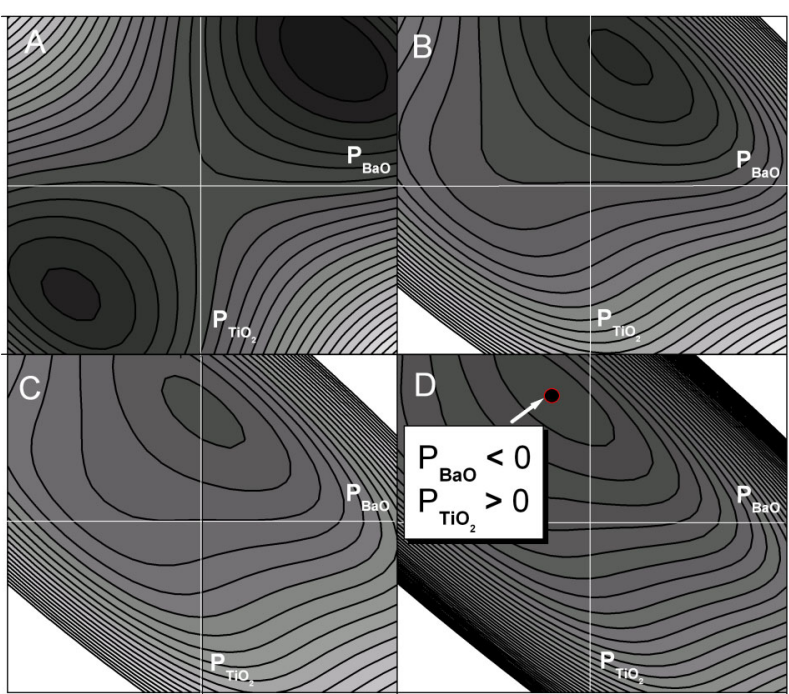

FIG. 2 (color online). Energy landscape $E^{\prime}\left(p_{\mathrm{TiO}_{2}}, p_{\mathrm{BaO}}\right)$ for different values of $\theta / M$ (film thickness). The interaction parameters for both interfaces were considered equal, $\theta_{R}=\theta_{L}=$ $\theta$, and were kept fixed as the film size $M$ is varied. The remaining parameters of the model have been derived from our $a b$ initio calculations. (a) $\theta / M=0$, (b) $\theta / M=3$, (c) $\theta / M=5$. For (d) $\theta / M=10$ the system displays a ferrielectric dipole pattern.

We estimate the interface parameters $\theta_{R}, \theta_{L}, p_{L}, p_{R}$ from our simulations [22] and we assume that their numerical values will not depend upon the film thickness (as observed in the actual $a b$ initio results, at least to first order).

As we vary the thickness of the ferroelectric film, we observe a change of the total energy landscape caused by the interplay between the different energetic contributions. This is clearly seen in Fig. 2, where we show contour plots of $E^{\prime}\left(p_{\mathrm{BaO}}, p_{\mathrm{TiO}_{2}}\right)$ for different values of $M$. For thick films, the spatial distribution of dipoles that minimizes the total energy forms a domain where both LPs in the $\mathrm{BaO}$ and $\mathrm{TiO}_{2}$ planes have the same sign [Fig. 2(a)]. As the number of unit cells is lowered, the minimum shifts to lower values of $p_{\mathrm{BaO}}$ until at a critical thickness [Fig. 2(b)] it becomes zero. Further reduction of the number of layers $M$ flips only the dipoles belonging to the $\mathrm{BaO}$ planes establishing a FDP [see Fig. 2(d)].

In summary, by exploiting the concept of layer polarization in the description of ferroelectric thin films between metal contacts, we have shown that the ferroelectric response of a thin film is indeed critically influenced by the interface properties of the system and that the analysis of the local variation of layer polarization captures completely the physical characteristics of the ferroelectric. Moreover, these results suggest the possibility that a FDP could be the normal state of a ferroelectric thin film at the nanoscale, even combined with the formation of twodimensional island domains. It would be tempting to link the formation of such FDPs to the appearance or not of a two-dimensional island below the critical thickness, and it would be desirable to understand its ultimate dependence upon the detailed interface structure and the nature of the metal contact. However, more studies are needed to explore these hypotheses.

The authors wish to acknowledge discussions with Arrigo Calzolari, Andrea Ferretti, and Mara Granada. We also thank Marco Fornari for his critical reading of the manuscript. This work has been supported in part by NSF and BES DOE. Calculations have been carried out at NCCS-ORNL and NCSU-HPC.

[1] C. H. Ahn, K. M. Rabe, and J.-M. Triscone, Science 303, 488 (2004).

[2] N. Spaldin, Science 304, 1606 (2004).

[3] J. Junquera and P. Ghosez, Nature (London) 422, 506 (2003).

[4] C. G. Duan, R. F. Sabirianov, W. N. Mei, S. S. Jaswal, and E. Y. Tsymbal, Nano Lett. 6, 483 (2006).

[5] G. Gerra, A. K. Tagantsev, N. Setter, and K. Parlinski, Phys. Rev. Lett. 96, 107603 (2006).

[6] N. Sai, A. M. Kolpak, and A. M. Rappe, Phys. Rev. B 72, 020101(R) (2005).

[7] P. Ghosez and J. Junquera, arXiv:cond-mat/0605299, and references therein.

[8] R. McKee, F. Walker, M. Buongiorno Nardelli, W. A. Shelton, and G. M. Stocks, Science 300, 1726 (2003).

[9] R. Resta, Rev. Mod. Phys. 66, 899 (1994).

[10] R. D. King-Smith and D. Vanderbilt, Phys. Rev. B 47, 1651 (1993).

[11] N. Marzari and D. Vanderbilt, Phys. Rev. B 56, 12847 (1997).

[12] F. Giustino, P. Umari, and A. Pasquarello, Phys. Rev. Lett. 91, 267601 (2003).

[13] F. Giustino and A. Pasquarello, Phys. Rev. B 71, 144104 (2005).

[14] X. Wu, O. Dieguez, K. M. Rabe, and D. Vanderbilt, Phys. Rev. Lett. 97, 107602 (2006).

[15] M. Nunez and M. Buongiorno Nardelli, Phys. Rev. B 73, 235422 (2006).

[16] S. Baroni et al., computer code PWSCF, http://www. pwscf.org).

[17] A. Ferretti et al., computer code WANT, http://www. wannier-transport.org); A. Calzolari, N. Marzari, I. Souza, and M. Buongiorno Nardelli, Phys. Rev. B 69, 035108 (2004).

[18] I. Souza, N. Marzari, and D. Vanderbilt, Phys. Rev. B 65, 035109 (2001).

[19] The description of the ferroelectric state in term of Wannier centers as "static" charges is equivalent to a direct mapping of the quantum mechanical problem of evaluating polarization in solids onto an effective classical one; see D. Vanderbilt and R. D. King-Smith, Phys. Rev. B 48, 4442 (1993) and Ref. [14] for more details.

[20] R. E. Cohen, Nature (London) 358, 136 (1992).

[21] S. Baroni, M. Peressi, R. Resta, and A. Baldereschi, in Proceedings of the Twenty-First International Conference on The Physics of Semiconductors, Beijing, 1992, edited by Ping Jiang and Hou-Zhi Zheng (World Scientific, Singapore, 1993), p. 689.

[22] A more detailed description of the model and its parameters will be published elsewhere. 